\title{
Changes in Optical Conductivity due to Readjustments in Electronic Density of States
}

\author{
Mei-Rong $\mathrm{Li}^{1}$ and J. P. Carbotte ${ }^{2}$ \\ ${ }^{1}$ Department of Physics, University of Guelph, Guelph, Ontario, Canada N1G 2W1 \\ ${ }^{2}$ Department of Physics and Astronomy, McMaster University, Hamilton, Ontario, Canada L8S $4 M 1$
}

\begin{abstract}
Within the model of elastic impurity scattering, we study how changes in the energy dependence of the electronic density of states (EDOS) $N(\epsilon)$ around the Fermi energy $\epsilon_{F}$ are reflected in the frequency-dependent optical conductivity $\sigma(\omega)$. While conserving the total number of states in $N(\epsilon)$ we compute the induced changes in $\sigma(\omega)$ as a function of $\omega$ and in the corresponding optical scattering rate $1 / \tau_{\text {op }}(\omega)$. These quantities mirror some aspects of the EDOS changes but the relationship is not direct. Conservation of optical oscillator strength is found not to hold, and there is no sum rule on the optical scattering rate although one does hold for the quasiparticle scattering. Temperature as well as increases in impurity scattering lead to additional changes in optical properties not seen in the constant EDOS case. These effects have their origin in an averaging of the EDOS around the Fermi energy $\epsilon_{F}$ on an energy scale set by the impurity scattering.
\end{abstract}

PACS numbers: 78.20.Bh, 78.20.-e

\section{INTRODUCTION}

Measurements of the infrared conductivity $\sigma(\omega)$ as a function of energy $\omega$, continue to give valuable information on charge dynamics in a wide range of metallic systems including the high- $T_{c}$ superconducting cuprates岾. These materials have received a lot of recent attention because they represent strongly correlated systems which exhibit new physics, beyond the usual Fermi liquid (FL) description of electric structure. It was recognized and emphasized as crucial very early on, that the normal state properties of the cyprates are anomalous. A Marginal Fermi liquid (MFL) 1 , which could describe remarkably well many of the observed deviation from FL behavior of the normal state. An essential feature of the MFL is that quasiparticle weight in the single particle charge carrier spectral density denoted by $Z^{-1}$ goes to zero logarithmically as the Fermi energy is approached. In this limit, there are no well defined quasiparticle poles, and the entire spectral density consists of an incoherent background which is due to the interactions. It is the delta function-like quasiparticle contribution (broadened by the interaction) which leads 胡 a Drude like contribution in the optical conductivity 6 . The incoherent background is responsible for the Holstein tails due, for example, to phonon assisted absorption in the well studied case of the electronphonon interaction. The incoherent contribution to the optical conductivity gives additional information on correlation effects complementary to the Drude response. Both contributions are described microscopically by the electron self energy $\Sigma(\omega)$ vs $\omega$, which is the fundamental quantity about which we would like information from measurements on the optical conductivity $\sigma(\omega)$. For example, the real part $\left(\Sigma_{1}\right)$ of $\Sigma$ deals with mass renormalization of the quasiparticles (qps) and the imaginary part $\left(\Sigma_{2}\right)$ is related to their lifetimes. As we have just described, $\Sigma$ can also lead to an incoherent background.

One of the most striking manifestation of correlation effects in the high- $T_{c}$ superconducting cuprates are the pseudogap features observed in their normal state. They are particularly prominent in underdoped systems, but are also known to be present at optimum dopingl The precise origin of the pseudogap is not yet known and this remains a controversial area. Nevertheless, the experimental situation is reasonably well characterized and has been reviewed by Timusk and Statte.

The pseudogap has been identified as a distinctive and sometimes even abrupt change in the temperature variation of the nuclear spin lattice relaxations, of the Knight shift 0 , of the dc resistivity 10.11 , and of the specific heat 12.13 .14 , in the frequency dependence of the infrared conductivity 15 and the current voltage characteristics of a tunneling junction 16 as angll as in angular resolved photo emission (ARPES) 17, L2, 29 . This last experimental technique is particularly powerful and has revealed that the pseudogap is not constant around the Fermi surface. Rather it has a $d$-wave nature which is the same symmetry as is exhibited by the superconducting gap below $T_{c}$ in the cuprates.

That the pseudogap has its origin in correlation effects is not in doubt. Rather, the issue is how it is to be

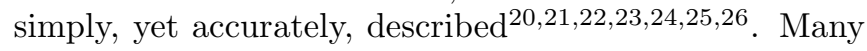
theoretical suggestions have been made. One widely held view is based on the so-called preformed pair model in which it is envisioned that the Cooper pairs exist above $T_{c}$ up to a higher pseudogap temperature $T^{*}$, but without phase coherence. The phase coherence between the pairs which is essential for superconductivity, sets in only at lower temperature $T<T_{c} 23$. 4 . In another model, different but related, finite momentum pairs are believed to be responsible for the pseudogap feature 25 . A very differentrecent proposal is the suggestion of Chakravarty et al.26 of $D$-density wave formation with attended orbital currents which double the crystallographic unit cell. There are also proposals encoded in the ideas of spincharge separation 2122 and the pioneering suggestion of Anderson 20.

The true nature of the changes that are brought about in the energy dependent electronic density of states 
(EDOS) $N(\epsilon)$ by the formation of the pseudogap remains unknown other than that the EDOS is depressed in some way. Consequently we will not address this specific case directly here although it is a motivating force for what we have done. Instead we will be concerned with a related but less specific issue, namely, the general question of how changes in $N(\epsilon)$ around the Fermi energy will manifest themselves in corresponding changes in the frequency dependence of the optical conductivity $\sigma(\omega)$ vs $\omega$. After all, from an experimental point of view, it is important to understand what qualitative signature is to be looked for which correspond to microscopic changes in $N(\epsilon)$.

To remain as simple as possible, we will examine in this paper in some detail mainly a simple model for $N(\epsilon)$ which consists of a constant background $N_{b}$ modified by two Lorentzian forms, both chosen to be symmetric about the Fermi energy. This assumption allows us to take advantage of the mathematical simplifications associated with the existence of particle-hole symmetry. In addition, one of the Lorentzian form is taken to add states to $N_{b}$, while the other subtracts states so that there is conservation of total number of states when $N(\epsilon)$ is integrated over energy, i.e., $\int_{-\infty}^{\infty} \Delta N(\epsilon) d \epsilon=0$, where $\Delta N(\epsilon)$ is the change in the EDOS. The Lorentzian form has the important simplifying property that an energy integral in the definition of the conductivity can be done analytically.

For simplicity we also limit ourselves to the case of elastic impurity scattering. This case has been extensively studied in the approximation that $N(\epsilon)$ is constant in the energy range about the Fermi energy which is significant for transport. For a constant $N_{b}$ the quasiparticle scattering rate $1 / \tau_{\mathrm{qp}}(\omega) \equiv-\Sigma_{2}(\omega)$ is constant independent of energy $\omega$. The conductivity takes on the well-known Drude form with constant transport scattering rate which gives the half width of the Drude and is in fact equal to twice the quasiparticle scattering rate. When inelastic scattering is considered, the quasiparticle scattering rate can still be defined in terms of the self energy $\Sigma_{2}(\omega)$, but nomf it acquires a temperature and

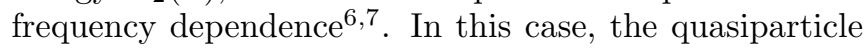
scattering time $\tau_{\mathrm{qp}}(\omega)$ is no longer equal to the optical scattering time $\tau_{\mathrm{op}}(\omega)$, which is formally defined in terms of $\sigma(\omega)=\sigma_{1}(\omega)+i \sigma_{2}(\omega)$ through the formula

$$
\frac{1}{\tau_{\mathrm{op}}(\omega)}=\frac{\Omega_{p}^{2}}{4 \pi} \operatorname{Re} \frac{1}{\sigma(\omega)}=\frac{\Omega_{p}^{2}}{4 \pi} \frac{\sigma_{1}(\omega)}{\sigma_{1}^{2}(\omega)+\sigma_{2}^{2}(\omega)},
$$

where $\Omega_{p}$ is the plasma frequency which is related to the real part of the conductivity $\sigma_{1}(\omega)$ through the optical oscillator strength sum rule

$$
\int_{0}^{\infty} d \omega \sigma_{1}(\omega)=\frac{\Omega_{p}^{2}}{8}
$$

In contrast to the constant EDOS case, when $N(\epsilon)$ varies with $\epsilon$ around the Fermi energy, $1 / \tau_{\mathrm{qp}}$ and $1 / \tau_{\mathrm{op}}$ are no longer constant just as in the inelastic case and are not equal. Each acquires a separate dependence on energy. The imaginary part of the electron self energy $\Sigma_{2}(\omega)$ becomes proportional to the self-consistent quasiparticle density of states $\tilde{N}(\omega)$ of the impure system. Impurities broaden the pure crystal EDOS $N(\epsilon)$ leading to $\tilde{N}(\omega)$. The optical scattering time defined in Eq. (11) also acquires $\omega$ dependence, and can be quite different from the quasiparticle scattering time 6 , which is measured in ARPES experiments. Thus, optical and ARPES data give complementary information on $\Sigma(\omega)$. What is measured in ARPES is the single particle spectral density for a particular momentum $\mathbf{k}$ as a function of $\omega$. It is denoted by $A(\mathbf{k}, \omega)$ and is related to the self energy $\Sigma(\omega)$, with $\mathbf{k}$ dependence suppressed, by

$$
A(\mathbf{k}, \omega)=-\frac{1}{\pi} \frac{\Sigma_{2}(\omega)}{\left[\omega-\epsilon_{\mathbf{k}}-\Sigma_{1}(\omega)\right]^{2}+\Sigma_{2}^{2}(\omega)} .
$$

The interpretation of optical results is now no longer straightforward. As an example of the complications that arise we note that for the case (as we will assume in most of our calculations here) when $N(\epsilon)$ conserves states, the integral over energy of the ARPES rate will also remain unchanged. This is because $1 / \tau_{\mathrm{qp}}(\omega)$ is proportional to $\tilde{N}(\omega)$ and $\int_{-\infty}^{\infty} \Delta \tilde{N}(\epsilon) d \epsilon=0$ is guaranteed when $\int_{-\infty}^{\infty} \Delta N(\epsilon) d \epsilon=0$. This sum rule however does not hold for $1 / \tau_{\mathrm{op}}(\omega)$, as have been previously discussed 27 for the case of inelastic scattering processes and for the onset of superconductivity, with constant EDOS. Nor is the total optical oscillator strength defined in Eq. (2) constant. This arises because the integral over $\sigma_{1}(\omega)$ defining $\Omega_{p}$ depends on an average of the EDOS $N(\epsilon)$ around the Fermi energy over an energy scale defined by the impurity scattering and is not just dependent on $N(0)$ as it would be in the familiar constant EDOS case. There is also an attendant temperature dependence of $\Omega_{p}$. As the temperature becomes comparable to the energy scale on which $N(\epsilon)$ varies significantly, the energy dependence in $N(\epsilon)$ is effectively smeared out and we recover a simple Drude form.

The energy dependent EDOS enters the formula for the conductivity in two places. First, the total current is the sum of the partial currents contributed by each state $|\mathbf{k}\rangle$ in the electron system. When this sum is changed into an integral over energy a first factor of $N(\epsilon)$ enters. But there is a second factor of $N(\epsilon)$ that also comes in from the quasiparticle scattering rate. This rate is proportional to the matrix element of the impurity potential which is to be averaged over all final states in which the electron can scatter. We can call this a final state effect. This second factor enters the ARPES rate which becomes proportional to the self-consistent $\tilde{N}(\omega)$. Clearly, ARPES and optical rates can no longer simply be proportional to each other. Both the initial and final states factors modify the optical scattering rate.

In our calculations, we find that the factor of $N(\epsilon)$ coming from the sum over partial currents from each electron has less of an effect on the energy dependence of $\sigma_{1}(\omega)$ than does the modification of the underlying 
ARPES rate due to final states effects. For a model of $N(\epsilon)$ which has a depression in the EDOS at $\epsilon=\epsilon_{F}$, which is, of course, compensated for at higher energies so as to conserve the total number of states, the first factor of $N(\epsilon)$ decreases the dc conductivity more than at finite frequency so that the overall effect is to lead to an apparent broadening of the Drude-like form for $\sigma_{1}(\omega)$. On the other hand, the ARPES rate is effectively reduced at small $\omega$ by the final state factor of $\tilde{N}(\epsilon)$. This sharpens the Drude-like line at small $\omega$. Thus the two effects have opposite tendencies, compete against each other and partially cancel. In the specific cases considered, the modifications in $\sigma_{1}(\omega)$ brought about by the changes in the ARPES rate are more important.

In a final set of calculations we also consider the case of a step-function EDOS model. In a metal $N(\epsilon)$ is expected to be finite at the Fermi energy although it could be small as compared to its value away from $\epsilon=\epsilon_{F}$. With our step model we show that a small but finite value $h$ of $N(\epsilon)$ for $|\epsilon|<$ some energy $E_{g}$ about $\epsilon=0$ always leads to the existence of Drude-like peak in the optical response, in sharp contrast to the case $h=0$ when a gap forms and the Drude peak is completely eliminated.

The paper is organized as follows. In Sec. II, we present a general theory of the optical conductivity in the case of impurity scattering. The simplest Drude limit is discussed in Sec. III as a reference. Sec. IV is devoted to a discussion of the effect of energy-dependent EDOS on optical conductivity, within a toy model for the EDOS involving two Lorentzian forms. This is followed by a parallel discussion, in Sec. V, for another EDOS model, the step model, which allows us to contrast metallic-like and semiconducting-like behavior. Finally, Sec.VI contains our conclusion. Some mathematics is shown in Appendix A and B.

\section{GENERAL THEORY FOR OPTICAL CONDUCTIVITY}

In linear response theory, the optical conductivity can be expressed as

$$
\sigma(\omega)=\frac{i}{\omega}[\Pi(\omega+i \delta)-\Pi(i \delta)],
$$

where $\Pi(\omega+i \delta)$ is the retarded polarization function. Under the assumptign that vertex corrections are negligible, $\Pi(\omega+i \delta) \operatorname{read} 29$

$$
\begin{gathered}
\Pi(\omega+i \delta)=\frac{\Omega_{p 0}^{2}}{4 \pi} \int_{-\infty}^{\infty} d \epsilon \frac{N(\epsilon)}{N_{b}} \int_{-\infty}^{\infty} d x f(x) A(\mathbf{k}, x) \\
\times[G(\epsilon, x+\omega+i \delta)+G(\epsilon, x-\omega-i \delta)]
\end{gathered}
$$

where $N(\epsilon) / N_{b}$ is the normalized EDOS with $N_{b}$ the constant background EDOS, $f(x)$ the Fermi distribution function, $G(\epsilon, x \pm i \delta)$ the quasiparticle Green function, and $\Omega_{p 0}$ the bare plasma frequency which, for an energydependent EDOS, will be shown below to be different from the real plasma frequency $\Omega_{p}$ defined in Eq. (2). $A(\mathbf{k}, \omega)$ is the spectral density defined in Eq. (3). It is important at this point to emphasize that although, as we have stated, we have neglected corrections to the electromagnetic vertex, the bare vertex itself can introduce further complications in Eq. (5). The EDOS factor $N(\epsilon)$ appearing in this equation comes from the conversion of a sum over all electron momenta into an integration over energy. But there is also a factor of the square of the Fermi velocity which is the electromagnetic vertex in our work, and this factor can have energy dependence. As we will not evaluate $N(\epsilon)$ or for that matter the electron velocity $v(\epsilon)$ from first principles but rather simply use a Lorentzian model, we can think that our model for the EDOS already contains the Fermi velocity and any dependence it may have on energy $\epsilon$. There is one caution we should make however. Later we will see, in our discussion of the quasiparticle scattering rate, that a second factor of the EDOS enters and this one is not multiplied by the electron velocity squared. This second factor will further get renormalized by the impurity scattering, and so is replaced everywhere by the dressed $\operatorname{EDOS} N(\omega)$. This should allow us to distinguish between these two factors of EDOS, and we will not emphasize this complication further but it should be kept in mind.

In the case of elastic scattering with no momentum dependence (no anisotropy), $G(\epsilon, x)$ can be written as

$$
\begin{aligned}
G(\epsilon, x \pm i \delta) & =G_{1}(\epsilon, x) \mp i \pi A(\epsilon, x) \\
& =\left[x-\epsilon-\Sigma_{1}(x) \pm i g(x)\right]^{-1},
\end{aligned}
$$

where

$$
G_{1}(\epsilon, x)=\frac{x-\Sigma_{1}(x)-\epsilon}{\left[x-\Sigma_{1}(x)-\epsilon\right]^{2}+g^{2}(x)},
$$

and $g(x)=\left|\Sigma_{2}(x)\right|=\tau_{\mathrm{qp}}^{-1}(x)$ the quasiparticle scattering rate. $\Sigma_{1}(x)$ and $g(x)$ satisfy the following KramersKronig (KK) relation

$$
\Sigma_{1}(x)=-\frac{1}{\pi} \int_{-\infty}^{\infty} d x^{\prime} \frac{g\left(x^{\prime}\right)}{x^{\prime}-x} .
$$

Inserting Eq. (5) into Eq. (4) leads to

$$
\begin{aligned}
& \sigma_{1}(\omega)=\frac{\Omega_{p 0}^{2}}{4 \pi} \pi \int_{-\infty}^{\infty} d \epsilon \frac{N(\epsilon)}{N_{b}} \int_{-\infty}^{\infty} \frac{d x}{\omega} f(x) A(\epsilon, x) \\
& \times[A(\epsilon, x+\omega)-A(\epsilon, x-\omega)] \\
& \sigma_{2}(\omega)=\frac{\Omega_{p 0}^{2}}{4 \pi} \int_{-\infty}^{\infty} d \epsilon \frac{N(\epsilon)}{N_{b}} \int_{-\infty}^{\infty} \frac{d x}{\omega} f(x) A(\epsilon, x) \\
& \times\left[G_{1}(\epsilon, x+\omega)+G_{1}(\epsilon, x-\omega)-2 G_{1}(\epsilon, x)\right],
\end{aligned}
$$

Eqs. (9) and (10) show how the two effects of the energydependent EDOS mentioned in the Introduction enter: one is the factor $N(\epsilon)$ coming from the sum over partial currents from each electron in the Fermi sea, the other arises from the electron spectral density factor $A(\mathbf{k}, \omega)$, 
which contains a factor of the final states the particles are scattered into.

The real and imaginary part of the conductivity, $\sigma_{1}$ and $\sigma_{2}$, respectively, obey the $\mathrm{KK}$ relation, namely,

$$
\sigma_{2}(\omega)=-\frac{2}{\pi} \omega \int_{0}^{\infty} d \omega^{\prime} \sigma_{1}\left(\omega^{\prime}\right) \mathrm{P} \frac{1}{\omega^{\prime 2}-\omega^{2}} .
$$

Eqs. (11) and (2) lead to the useful relationship,

$$
\lim _{\omega \rightarrow \infty} \sigma_{2}(\omega)=\frac{1}{\omega} \frac{\Omega_{p}^{2}}{4 \pi} .
$$

At zero T, Eq. (9) simplifies greatly and becomes

$$
\begin{aligned}
\sigma_{1}(\omega)= & \frac{\Omega_{p 0}^{2}}{4 \pi} \pi \int_{-\infty}^{\infty} d \epsilon \frac{N(\epsilon)}{N_{b}} \int_{-\omega}^{0} \frac{d x}{\omega} \\
& \times A(\epsilon, x) A(\epsilon, x+\omega) .
\end{aligned}
$$

The dc conductivity immediately reads

$$
\begin{aligned}
\sigma(0) & =\frac{\Omega_{p 0}^{2}}{4 \pi} \pi \int_{-\infty}^{\infty} d \epsilon \frac{N(\epsilon)}{N_{b}} A^{2}(\epsilon, 0) \\
& =\frac{\Omega_{p 0}^{2}}{4 \pi} \frac{1}{\pi} \int_{-\infty}^{\infty} d \epsilon \frac{N(\epsilon)}{N_{b}} \frac{g^{2}(0)}{\left[\epsilon^{2}+g^{2}(0)\right]^{2}}
\end{aligned}
$$

In Eq. (14) we have used $\Sigma_{1}(0)=0$.

We assume that the impurity potential $V$ is small and thus the impurity scattering can be treated within the Born approximation. Within the non-selfconsistent (nsc) Born approximation, the self energy reads

$$
\begin{aligned}
\Sigma_{\mathrm{nsc}}^{(\mathrm{ret})}(\omega) & =\Sigma_{1 \mathrm{nsc}}(\omega)-i g_{\mathrm{nsc}}(\omega) \\
& =\gamma_{0} \int_{-\infty}^{\infty} d \epsilon \frac{N(\epsilon)}{N_{b}} \frac{1}{\omega-\epsilon+i 0^{+}}
\end{aligned}
$$

where $\gamma_{0}=n_{i} V^{2} N_{b}$ with $n_{i}$ the impurity density.

For an energy-dependent EDOS, the selfconsistent (sc) Born approximation gives instead

$$
\begin{aligned}
\Sigma_{\mathrm{sc}}^{(\mathrm{ret})}(\omega) & =\Sigma_{1 \mathrm{sc}}(\omega)-i g_{\mathrm{sc}}(\omega) \\
& =\gamma_{0} \int_{-\infty}^{\infty} d \epsilon \frac{N(\epsilon)}{N_{b}} G\left(\epsilon, \omega+i 0^{+}\right),
\end{aligned}
$$

where the full selfconsistent $G$ appears on the right-hand side of Eq. (16), so this equation must be solved by successive iteration until convergence is achieved.

Before leaving this section we emphasize that the factor $N(\epsilon)$ that enters Eqs. (15) and (16) is the EDOS when no impurities are present and comes from conversion of a sum over momentum to one over energy. It does not include any additional electron velocity factor $v(\epsilon)$. This is to be contrasted with the formula for the conductivity (Eqs. (4) and (5)), in which we have suppressed a factor of $v(\epsilon)^{2}$, and so the EDOS factor that enters that formula should further contain any energy dependence there might be in $v(\epsilon)^{2}$. In our discussion below, we will describe the effect on the conductivity of each of these two factors separately. Note that $g_{\mathrm{sc}}(\omega)$ in Eq. (16) is directly proportional to the renormalized $\operatorname{EDOS} \tilde{N}(\omega)$ given by $\tilde{N}(\omega)=-\int d \epsilon\left[N(\epsilon) / N_{b}\right] A(\epsilon, \omega)$.

\section{DRUDE FORMULA FOR CONSTANT EDOS}

In the case of a constant EDOS $N(\epsilon) \equiv N_{b}$, both the non-selfconsistent and selfconsistent Born approximation lead to $\Sigma_{1} \equiv 0, g(\omega) \equiv \pi \gamma_{0}=\Gamma / 2$. Eqs. (9) and (10) thus result in the well-known Drude formula

$$
\begin{aligned}
\sigma_{1}^{(\text {Drude })}(\omega) & =\frac{\Omega_{p 0}^{2}}{4 \pi} \frac{1 / \Gamma}{1+(\omega / \Gamma)^{2}} \\
\sigma_{2}^{(\text {Drude })}(\omega) & =\frac{\Omega_{p 0}^{2}}{4 \pi} \frac{\omega / \Gamma^{2}}{1+(\omega / \Gamma)^{2}}
\end{aligned}
$$

$\sigma_{1}^{(\text {Drude) }}(\omega)$ in Eq. (17) is a Lorentzian function of $\omega$ with the half-width $\Gamma$. When Eqs. (17) and (18) are substituted into Eq. (11) which defines the optical scattering rate, we get $\tau_{\text {op }}^{-1}(\omega)^{(\text {Drude })} \equiv 2 g(\omega)=\Gamma$. In this simple case, the optical scattering rate is just equal to twice the quasiparticle scattering rate. Thus optical experiments access directly the microscopic information on the imaginary part of the self energy. Further, Eq. (17) also gives the dc conductivity and the plasma frequency as

$$
\begin{aligned}
& \sigma^{(\text {Drude })}(0)=\frac{\Omega_{p 0}^{2}}{4 \pi} \frac{1}{\Gamma}, \\
& \Omega_{p}^{(\text {Drude })}=\Omega_{p 0} .
\end{aligned}
$$

$\sigma_{1}^{(\text {Drude })}(\omega)$ and $\tau_{\text {op }}^{-1}(\omega)^{\text {(Drude) }}$ as functions of $\omega$ are shown as green dot-dashed lines in Figs. 3, 4, 5 below and serve as a reference when we discuss the effects on the optical conductivity of an energy dependence in the EDOS.

\section{OPTICAL CONDUCTIVITY FOR EDOS WITH TWO LORENTZIAN FORMS}

Now we are in the position to study the influence of the energy dependence of EDOS on the optical conductivity. In this section, we consider the following model for EDOS,

$$
\frac{N(\epsilon)}{N_{b}}=1+\frac{s}{\pi}\left(\frac{a_{1}}{a_{1}^{2}+\epsilon^{2}}-\frac{a_{2}}{a_{2}^{2}+\epsilon^{2}}\right),
$$

where $s>0$. The two Lorentzian forms of Eq. (21) guarantee conservation of the total states: $\int_{-\infty}^{\infty} \Delta \vec{N}(\epsilon) d \epsilon=$ $\int_{-\infty}^{\infty}\left[N(\epsilon)-N_{b}\right] d \epsilon=0$. For $a_{1}>a_{2}$, there is a hole, i.e., depletion of states, around the Fermi surface. This is shown as the black solid line in Fig. 11. While $a_{1}<a_{2}$ corresponds to a peak, namely, additional states at and around the Fermi surface, as shown by the red dashed line in the same figure. The excess (missing) states are compensated for by a decrease (increase) in $N(\epsilon)$ at higher energies beyond $\epsilon=\sqrt{a_{1} a_{2}} \simeq 7$ in units of $\gamma_{0}$ for $a_{1}=5$, $a_{2}=10\left(a_{1}=10, a_{2}=5\right)$.

From Eqs. (15) and (21) we get the non-selfconsistent self energy based on the first-order Born approximation

$$
\Sigma_{1 \mathrm{nsc}}(\omega)=\gamma_{0} s\left(\frac{\omega}{\omega^{2}+a_{1}^{2}}-\frac{\omega}{\omega^{2}+a_{2}^{2}}\right),
$$




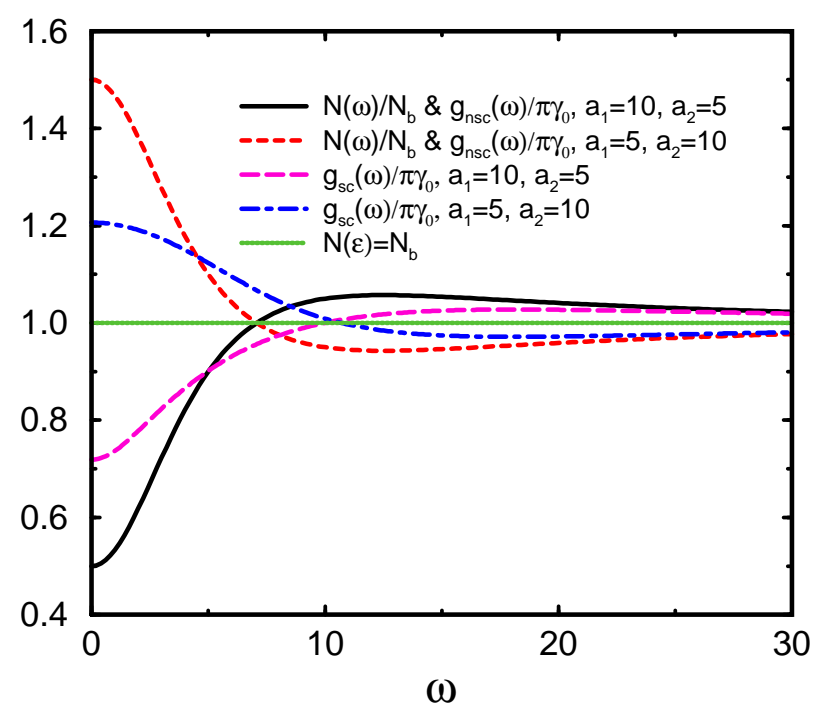

FIG. 1: Normalized density of states and ARPES scattering rate as functions of frequency in the Lorentzian-EDOS model shown in Eq. (21). We used $s=5 \pi$. All energies are in units of $\gamma_{0}$.

$$
\begin{aligned}
g_{\mathrm{nsc}}(\omega) & =\pi \gamma_{0} \frac{N(\omega)}{N_{b}} \\
& =\pi \gamma_{0}\left[1+\frac{s}{\pi}\left(\frac{a_{1}}{a_{1}^{2}+\epsilon^{2}}-\frac{a_{2}}{a_{2}^{2}+\epsilon^{2}}\right)\right]
\end{aligned}
$$

so the black solid line and the red dashed line shown in Fig. 11 also represent $g_{\mathrm{nsc}} / \pi \gamma_{0}$ vs $\omega$. Eqs. (16) and (21) yield the two equations determining the selfconsistent self energy

$$
\begin{aligned}
& \Sigma_{1 \mathrm{sc}}(\omega)=\gamma_{0} s\left\{\frac{\omega_{0}}{\omega_{0}^{2}+g_{a 1}^{2}}-\frac{\omega_{0}}{\omega_{0}^{2}+g_{a 2}^{2}}\right\} \\
& g_{\mathrm{sc}}(\omega)=\pi \gamma_{0}+\gamma_{0} s\left\{\frac{g_{a 1}}{\omega_{0}^{2}+g_{a 1}^{2}}-\frac{g_{a 2}}{\omega_{0}^{2}+g_{a 2}^{2}}\right\}
\end{aligned}
$$

where $\omega_{0}=\omega-\Sigma_{1 \mathrm{sc}}(\omega), g_{a 1}=g_{\mathrm{sc}}(\omega)+a_{1}$, and $g_{a 2}=$ $g_{\mathrm{sc}}(\omega)+a_{2}$. Eqs. (23) and (24) can be easily solved numerically. There exists an extensive literature on the effect of energy dependence of the EDQS 2 the elestron self energy and on other properties $31,32,33,34,35,36,37$. Some aspects of the superconducting state as well as normal state have been explored although much of the literature deals with a peak, while here we have emphasized a pseudogap. The imaginary part $g_{\mathrm{sc}}$ obtained from Eqs. (23) and (24) for $a_{1}>a_{2}$ and for $a_{2}>a_{1}$ are shown in Fig. 11 as magenta long-dashed line and blue dot-dashed line, respectively. Comparing $g_{\text {nsc }}$ and $g_{\mathrm{sc}}$ in Fig. 1, we see that selfconsistency smoothes out the ARPES rate because it is broadened out by impurity scattering. In fact, this rate is simply proportional to the fully renormalized EDOS $\tilde{N}(\epsilon)$ defined by Eq. (24) with factor $\pi \gamma_{0}$ left out. Eq. (24) has the same form as Eq. (21) with the broadening in the Lorentzian form, i.e., $a_{1}$ and $a_{2}$ replaced by $a_{1}+g_{\mathrm{sc}}(\omega)$ and $a_{2}+g_{\mathrm{sc}}(\omega)$, respectively. An iterative solution is required until convergence is reached. In Fig. 1 we see that for the case of the hole (peak) at the Fermi surface $N(\epsilon) / N_{b}$ at $\epsilon=0$ is 0.5 (1.5) and the application of selfconsistency changes these numbers to about 0.7 (1.2). The smearing due to the impurity scattering is considerable in the EDOS.

\section{A. Constant ARPES rate $g(\omega)=\Gamma / 2$ but energy-dependent $N(\epsilon)$}

As we mentioned in the Introduction, there are two effects of EDOS on the optical conductivity, one is from summing over partial currents. This shows up as the explicit factor of $N(\epsilon)$ in Eqs. (9) and (10), the other is the final-state effect entering in the ARPES rate which shows up in Eq. (24). To see clearly the different roles these two effects play, we first switch off the effect of energy-dependent ARPES rate, and replace it by a constant $g(\omega)=\Gamma / 2$. Correspondingly, $\Sigma_{1}=0$.

We focus on the $T=0$ case, because it is simplest and allows us to produce partially analytic results. Eqs. (13) and (10) immediately yield

$$
\begin{aligned}
\sigma_{1}(\omega) & =\frac{\Omega_{p 0}^{2}}{4 \pi} \frac{1}{\pi} \int_{-\infty}^{\infty} d \epsilon \frac{N(\epsilon)}{N_{b}} \mathcal{J}_{\Gamma / 2}^{(\mathrm{re})}\left(\frac{\omega}{\Gamma / 2}, \frac{\epsilon}{\Gamma / 2}\right) \\
\sigma_{2}(\omega) & =\frac{\Omega_{p 0}^{2}}{4 \pi} \frac{1}{\pi} \int_{-\infty}^{\infty} d \epsilon \frac{N(\epsilon)}{N_{b}} \mathcal{J}_{\Gamma / 2}^{(\mathrm{im})}\left(\frac{\omega}{\Gamma / 2}, \frac{\epsilon}{\Gamma / 2}\right),
\end{aligned}
$$

where

$$
\begin{aligned}
\mathcal{J}_{\gamma}^{(\mathrm{re})}(\tilde{\omega}, e)= & \frac{2}{\gamma^{2}} \frac{1}{\tilde{\omega}\left(\tilde{\omega}^{2}+4\right)}\{\arctan (\tilde{\omega}+e) \\
& \left.+\frac{1}{\tilde{\omega}} \ln \left[\frac{(\tilde{\omega}+e)^{2}+1}{e^{2}+1}\right]\right\}, \\
\mathcal{J}_{\gamma}^{(\mathrm{im})}(\tilde{\omega}, e)= & \frac{1}{\gamma^{2}} \frac{1}{\tilde{\omega}\left(\tilde{\omega}^{2}+4\right)}\left\{\ln \left[\frac{(\tilde{\omega}+e)^{2}+1}{e^{2}+1}\right]\right. \\
& \left.-\frac{4}{\tilde{\omega}} \arctan (\tilde{\omega}+e)+\frac{1}{e^{2}+1}\right\} .
\end{aligned}
$$

The expressions for the dc conductivity and plasma frequency become particularly simple and very revealing. From Eqs. (14) and (2) we get

$$
\begin{aligned}
& \sigma(0)=\frac{\Omega_{p 0}^{2}}{4 \pi} \frac{2}{\Gamma} \int_{0}^{\infty} d x \frac{N(x \Gamma / 2)}{N_{b}} Y_{0}(x), \\
& \frac{\Omega_{p}^{2}}{8}=\frac{\Omega_{p 0}^{2}}{8} \int_{0}^{\infty} d x \frac{N(x \Gamma / 2)}{N_{b}} Y_{1}(x),
\end{aligned}
$$

where

$$
Y_{0}(x)=\frac{2}{\pi} \frac{1}{\left(x^{2}+1\right)^{2}}, \quad Y_{1}(x)=\frac{2}{\pi} \frac{1}{x^{2}+1}
$$

We have plotted the two functions $Y_{0}(x)$ and $Y_{1}(x)$ in Fig. 2. Both peak at $x=0$, and decay rapidly on a scale of $\epsilon$ equal to a few times $\Gamma / 2$. For small $\Gamma$, both $\sigma(0)$ 
and $\Omega_{p}$ depend strongly on the EDOS at and around the Fermi surface. If $N(\epsilon \simeq 0)<N_{b}$, which is the case for $a_{1}>a_{2}, \sigma(0)<\sigma^{\text {(Drude) }}(0)$ and $\Omega_{p}<\Omega_{p 0}$ immediately follow. In the opposite case for $a_{1}<a_{2}$ in which $N(\epsilon \simeq 0)>N_{b}$, we get $\sigma(0)>\sigma^{\text {(Drude) }}(0)$ and $\Omega_{p}>\Omega_{p 0}$. Besides, the peak in $Y_{1}(x)$ is broader than that in $Y_{0}(x)$, implying that the dc conductivity and the plasma frequency $\sigma(0)$ and $\Omega_{p}$ do not scale with each other for energy-dependent EDOS. The region in energy around the Fermi energy that is most important in determining $\sigma(0)$ and $\Omega_{p}^{2}$ is given by $\Gamma$. If, as we have assumed so far, the scale for $\Gamma$ is much less than the energy scale that controls important variations in the EDOS which in our case is $\left(a_{1}, a_{2}\right)$ in the Lorentzian form, then it is mainly the value of $N(\epsilon)$ at $\epsilon=0$ which comes in. But when $\Gamma$ is of the same order as $\left(a_{1}, a_{2}\right)$, this is no longer the case and the details of the variations in $N(\epsilon)$ are importantly sampled. Finally when $\Gamma$ is much greater than $\left(a_{1}, a_{2}\right)$, it will be only the size of the background $N_{b}$ that matters. This is the limit in which we regain the simple Drude model of Eqs. (17) and (18).

It is easy to find from Eqs. (26) and (30) that $\lim _{\omega \rightarrow \infty} \sigma_{2}(\omega)=\Omega_{p}^{2} / 4 \pi \omega$, indicating the KK relation Eq. (11) is automatically obeyed as we expect. For a general value of $\omega, \sigma_{1}$ and $\sigma_{2}$ in Eqs. (25) and (26) need to be obtained numerically. Inserting results of Eqs. (25), (26) and (30) into Eq. (11) allows us to obtain the optical scattering rate. Results will be discussed in Sec. 3. C.

\section{B. Constant $N(\epsilon)$ but energy-dependent ARPES rate $g(\omega)$}

If instead we switch off the energy-dependent EDOS by replacing it with $N(\epsilon)=N_{b}$, but turn on the energy dependent ARPES rate $g(\omega)$ alone, we are able to see the second effect on $\sigma$, coming from the ARPES rate alone. We have referred to this aspect of the problem earlier as a final state effect. Since in the present work the self energy is momentum independent, it is convenient to first integrate over $\epsilon$ in Eqs. (13) and (10) at $T=0$. After some algebra we obtain

$$
\begin{aligned}
& \sigma_{1}^{(\mathrm{cons})}(\omega)=\frac{\Omega_{p 0}^{2}}{4 \pi} \frac{1}{\pi} \lim _{D \rightarrow \infty} \int_{-\omega}^{0} \frac{d x}{\omega} \frac{1}{g(x)} \\
& =\frac{\Omega_{p 0}^{2}}{4 \pi} \int_{-\omega}^{0} \frac{d x}{\omega} \frac{1}{g(x)} \frac{g_{+}+1}{\left(g_{+}+1\right)^{2}+\left(x_{0}-x_{+}\right)^{2}}, \\
& \sigma_{2}^{(\mathrm{cons})}(\omega)=\frac{\Omega_{p 0}^{2}}{4 \pi} \frac{1}{\pi} \lim _{D \rightarrow \infty} \int_{-\infty}^{0} \frac{d x}{\omega} \frac{1}{g(x)} \\
& \times\left[\sum_{i= \pm} \mathcal{F}^{(\mathrm{im})}\left(\tilde{D}, g_{i}, x_{0}, x_{i}\right)-\mathcal{F}_{0}^{(\mathrm{im})}\left(\tilde{D}, x_{0}\right)\right] \\
& =\frac{\Omega_{p 0}^{2}}{4 \pi} \int_{-\omega}^{0} \frac{d x}{\omega} \frac{1}{g(x)} \frac{x_{+}-x_{0}}{\left(g_{+}+1\right)^{2}+\left(x_{0}-x_{+}\right)^{2}}
\end{aligned}
$$

where $\tilde{D}=D / g(x), x_{0}=\left[x-\Sigma_{1}(x)\right] / g(x), g_{ \pm}=g(x \pm$ $\omega) / g(x), x_{ \pm}=\left[x \pm \omega-\Sigma_{1}(x \pm \omega)\right] / g(x)$, and $\mathcal{F}^{(\mathrm{re})}, \mathcal{F}^{(\mathrm{im})}$, and $\mathcal{F}_{0}^{(\mathrm{im})}$ defined in Eqs. A1, A3 in Appendix A.

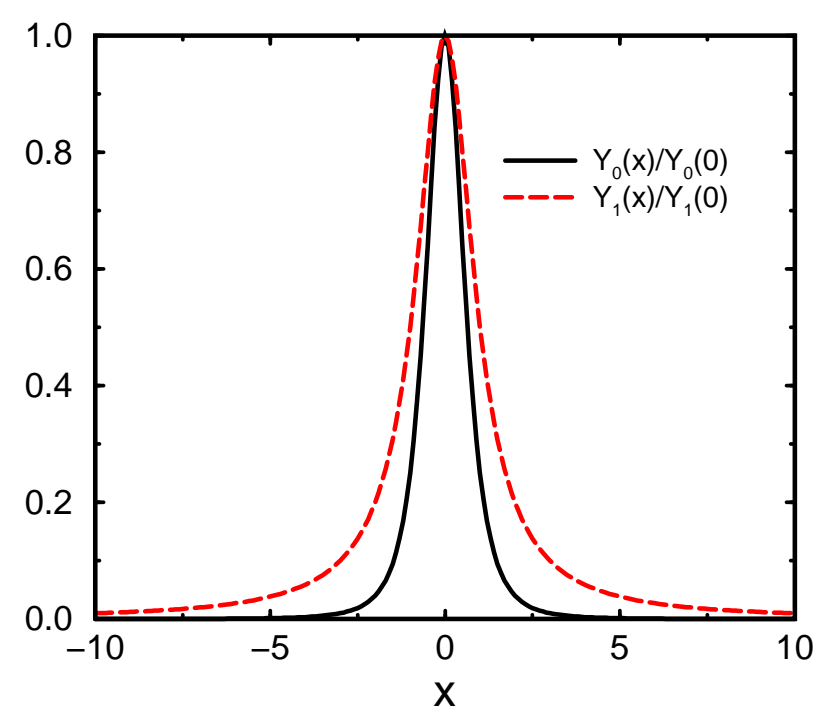

FIG. 2: Scaling functions $Y_{0}(x)=2 /\left[\pi\left(x^{2}+1\right)^{2}\right]$ and $Y_{1}(x)=$ $2 /\left[\pi\left(x^{2}+1\right)\right]$.

\section{Energy-dependent $N(\epsilon)$ and ARPES rate}

If we want to include in the calculations both the direct factor of $N(\epsilon)$ in Eq. (5) and the energy-dependent ARPES rate so as to study the competition between the initial-state effect and the final-state effect, we need to return to Eqs. (21) and (22) or (24) and insert these into Eqs. (13) and (11) to find $\sigma_{1}(\omega)$ and $\tau_{\text {op }}^{-1}(\omega)$. Note that the Lorentzian forms used for $N(\epsilon)$ allow us to do the integral over $\epsilon$ analytically, which greatly simplifies the numerical work. The expressions for $\sigma_{1}(\omega)$ and $\sigma_{2}(\omega)$ after integrating over $\epsilon$ are given in Appendix B. They are complicated but can easily be handled numerically.

Our first numerical results are presented in Fig. 3, which has two complementary frames (a) and (b). In all curves $a_{1}=10, a_{2}=5$ with $s=5 \pi$, in units of $\gamma_{0}$. This corresponds to a depression in the EDOS (See Eq. (21)) over its background value, as drawn in Fig. 1 solid black curve with $N(0)$ reduced by a factor of two compared to the background value. The missing states at small $\epsilon$ show up at larger $\epsilon$ and there is conservation of states by arrangement. Although electronic states are preserved this does not mean that the corresponding optical oscillator strength is, as we will see. In Fig. 3 (a) we show five curves. All give the real part of the optical conductivity $\sigma_{1}(\omega)$ in units of $\Omega_{p 0}^{2} / 4 \pi$ as a function of energy $\omega$ in units of $\gamma_{0}$. The first in green (dot-dashed) is for reference and is the usual Drude form (given in Eq. (17)) that obtains when the EDOS is constant as well as the elastic scattering rate. In this case, the width of the Drude is simply $2 \pi \gamma_{0} \equiv \Gamma$ and the oscillator sum rule 

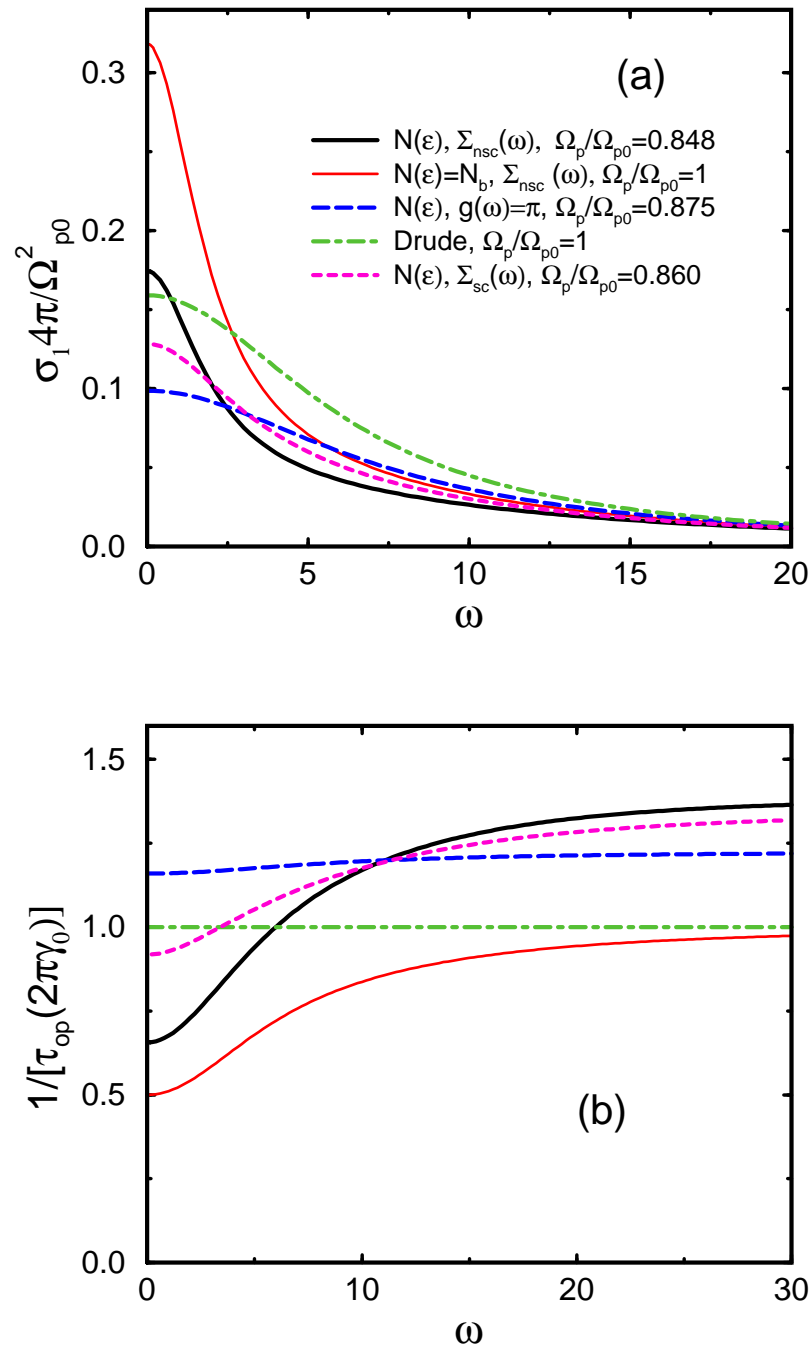

FIG. 3: (a) Real part of optical conductivity and (b) Optical scattering rate as functions of frequency in the LorentzianEDOS model shown in Eq. (21) with $a_{1}=10, a_{2}=5$ and $s=5 \pi$. We used $\gamma_{0}=1$. Labels of curves in (b) mean the same as in (a).

gives $\Omega_{p 0}^{2} / 8$. This no longer holds when energy dependence is included in the EDOS. Introduction of such a dependence in the theory leads to several modifications and we will take these in steps of added complications. The simplest modification is that an overall EDOS factor enters the sum over partial currents involving each participating electron. This was referred to previously as the sum over initial states. Including only this factor with a constant approximation for the scattering rate $g(\omega)=\pi$ in units of $\gamma_{0}$ gives the blue curve (long-dashed). The reduction in $N(\epsilon)$ at $\epsilon=0$ by a factor of 2 translates into a substantial reduction in $\sigma_{1}(\omega)$ at $\omega=0$ although by a factor that is substantially less than 2 . While the line shape is no longer perfectly Drude, its width at half maximum has increased over the Drude case $\Gamma=2 \pi \gamma_{0}$ and could lead one to conclude that the optical scattering 1 rate has increased. It is already clear from this remark that optical and quasiparticle scattering rate are no longer the same. In fact, this is shown explicitly in Fig. 3 (b). The green curve (dot-dashed) is constant but the blue curve (long-dashed) now exhibits a slight energy dependence and is everywhere larger than twice the quasiparticle rate. Another important modification brought about by the introduction of an energy dependence in $N(\epsilon)$ is that the optical sum rule defined in Eq. (2) as the sum over the real part of the conductivity has the plasma frequency reduced from $\Omega_{p 0}$ to $\Omega_{p}=0.875 \Omega_{p 0}$. Note that this occurs although no states are lost in $N(\epsilon)$. On the other hand, when the initial-state EDOS factor is taken to be independent of energy (constant $N_{b}$ ) there is no change in plasma frequency even if the quasiparticle scattering rate is energy dependent as shown in the red solid thin curve which was computed for constant $N(\epsilon)=N_{b}$, but with $\Sigma_{\text {nsc }}$ of Eq. (22) obtained in a nonselfconsistent theory for the quasiparticle scattering rate. By non-selfconsistent we mean a first iteration of the self energy equation in Eq. (16) as defined in Eq. (15). This modulates the scattering rate with precisely the same EDOS factor $N(\epsilon)$ that we used for the initial state sum in the blue curve. We see that in a real sense this factor has the opposite effect on the shape of the conductivity $\sigma_{1}(\omega)$ vs $\omega$ in that it increases its value at $\omega=0$, beyond what it is in the pure Drude case, and also effectively sharpens up the curve. This can be seen more quantitatively in Fig. 3 (b) where the red solid thin curve for the optical scattering rate falls everywhere below the green curve (dashed-dotted) of the Drude theory and also even further below the blue curve (long-dashed). Combining these two effects brings us back closer to our original Drude than including each separately, at low frequencies where the effects are biggest. This expectation is born out in the solid black curve which includes initial state $N(\epsilon)$ factor and non-selfconsistent quasiparticle scattering rate $g_{\mathrm{nsc}}(\omega)$. Note that the plasma frequency $\left(\Omega_{p} / \Omega_{p 0}=0.848\right)$ is not changed much from its value of 0.875 in the blue curve, which shows that the plasma frequency is mainly sensitive to the initial $N(\epsilon)$ and is less sensitive to the details of the quasiparticle scattering rate. The corresponding optical scattering rate is shown as the solid black curve in Fig. 月 (b). This curve contrasts greatly with the previous two curves blue (long-dashed) and red (solid thin). In both these curves there is no compensation in the scattering rate as compared to the Drude case in the sense that the blue curve is always above and the red curve always below. By contrast, the black curve (solid) is below at small $\omega$ and above at larger $\omega$. While there is some cancellation, no sum rule applies to the area under $1 / \tau_{\mathrm{op}}(\omega)$ when it is integrated over $\omega$. This represents a real difference between quasiparticle scattering rate and optical scattering rate since for the ARPES rate a sum rule does apply which is directly connected to the sum rule on $N(\epsilon)$. This shows clearly that, while optics can give microscopic information on scattering rates, it is not easy to relate the information so obtained, with the 
characteristics of the self energy which is, in the end, the fundamental quantity and is the quantity we would like to measure directly.
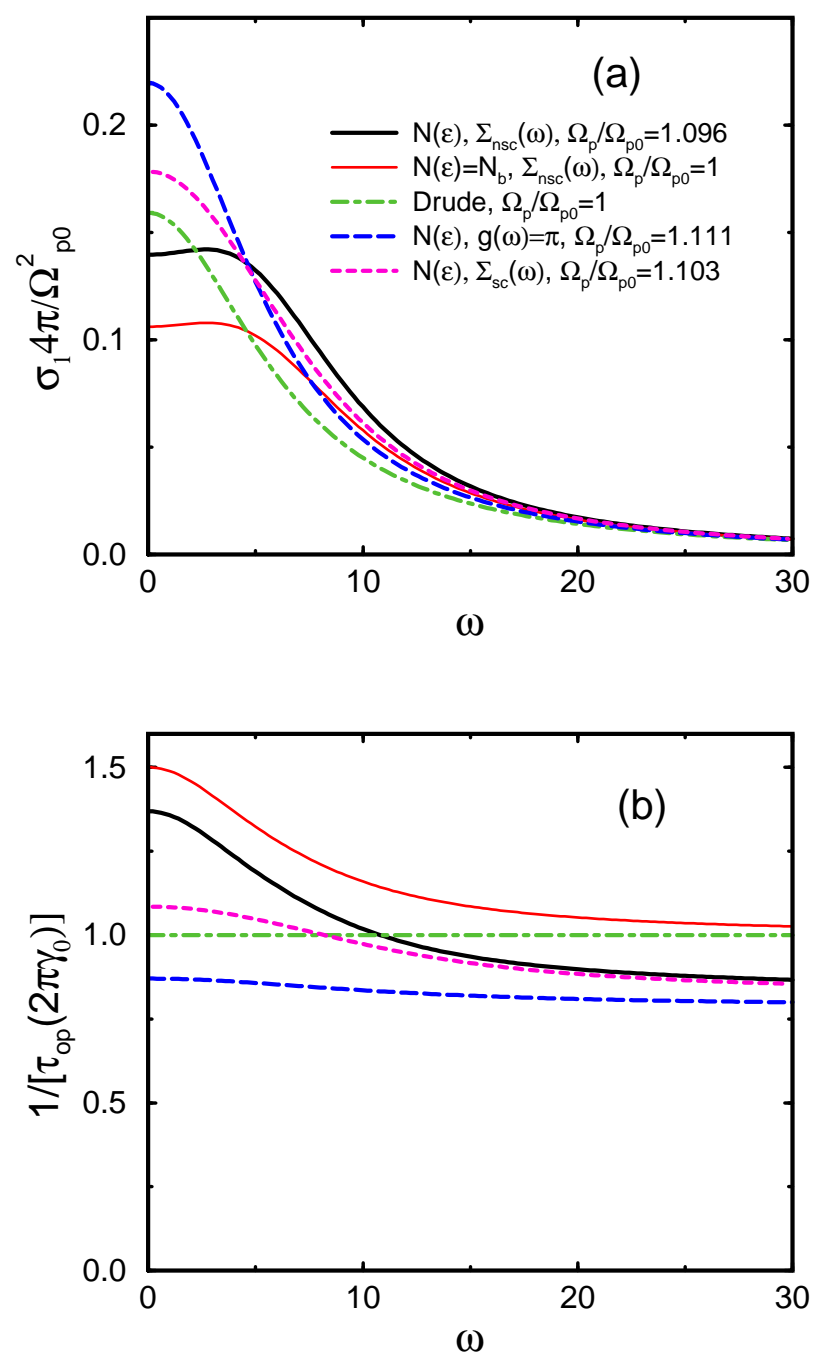

FIG. 4: (a) Real part of optical conductivity and (b) Optical scattering rate as functions of frequency in the LorentzianEDOS model shown in Eq. (21), with $a_{1}=5, a_{2}=10$ and $s=5 \pi$. We used $\gamma_{0}=1$. Labels of curves in (b) mean the same as in (a).

One final element of importance has been neglected so far. To get the effective scattering rate in a system with energy dependent EDOS with impurities, it is necessary to solve the self energy equation (16) selfconsistently through repeated iteration of Eqs. (23) and (24). Impurities will smear out a valley in $N(\epsilon)$ and change it to its selfconsistent value $\tilde{N}(\epsilon)$, which is what finally enters in the impurity scattering. When this is done we obtain a final curve for $\sigma_{1}(\omega)$ vs $\omega$, the magenta curve (dashed) of Fig. 3 (a) and for the optical scattering rate in Fig. 3 (b). As we would have expected, the self consistency smoothes out the curves but this does not introduce any new physics. The value of the plasma frequency is also not changed much over its non-selfconsistent value.

Before moving on to the case of a peak in the EDOS at the Fermi energy, we bring up once more the complication that arises from the electromagnetic vertex which introduces a product of two electron velocity $v(\epsilon)^{2}$ in the formula for the conductivity which effectively introduces a further energy dependence in the factor of $N(\epsilon)$ appearing directly in Eq. (4). This factor is not present in the self energy Eqs. (15) (non-selfconsistent) and (16) (selfconsistent). This means that the magenta (dashed) curve of Fig. 3 (a) could be further modified through the introduction of a $v(\epsilon)^{2}$ factor in our model $N(\epsilon)$. If we look at band structure calculations, we note that the product of $N(\epsilon) v(\epsilon)^{2}$ is often less dependent on energy than is $N(\epsilon) 30$. This means that in this case, the final results for the conductivity might move some way towards the results of a selfconsistent theory for the self energy with constant $N(\epsilon)$ as the explicit factor of EDOS in Eq. (14). One curve shown in Fig. 3 (a) is for $N(\epsilon)$ in Eq. (14) equal to a constant with non-selfconsistent self energy (red dotted line). The selfconsistent case would be smoothed out a little more as compared with this curve. Of course, in this work, we do not wish to tie ourselves to band structure calculations but rather treat a general model for the energy dependence.

In Fig. 4 we show results when there is a peak in $N(\epsilon)$ rather than a depression or gap. No new physics is associated with these curves. In a sense, the effects are opposite of those described in some detail for the previous case. For example, comparison of the results of full calculations including the self energy computed selfconsistently, shows that $\sigma_{1}(\omega)$ is above the simple Drude curve reflecting the fact that there is a peak in $N(\epsilon)$ centered at $\epsilon=0$, which enhances the value of $\sigma_{1}(\omega)$ around $\omega=0$, because there are more electronic states around the Fermi energy. Also when this same effect is included in the scattering process the scattering rate is increased at small $\omega$ as compared to its Drude value, which makes the curve $\sigma_{1}(\omega)$ vs $\omega$ look broader. In the example considered, the actual frequency dependence of the selfconsistent optical scattering rate is not very large (see the magenta dashed curve in Fig. (b) ) and its variation is smaller than found for the self-consistent quasiparticle scattering rate shown as the blue dot-dashed line in Fig. 11. These two curves are similar but are not the same in detail. In particular, we stress again that there is a sum rule which holds for the quasiparticle rate as compared to its Drude counterpart, i.e., no such sum rule applied to the area under the optical rate.

Another consequence of the energy dependence in $N(\epsilon)$ is that the conductivity will change with temperatures, an effect which does not arise in the ordinary Drude case. This is illustrated in Fig. 5 where we show the real part of the conductivity $\sigma_{1}(\omega)$ vs $\omega$ for three cases. The black solid curve repeats our previous results for the case of a gap at the Fermi energy and nonselfconsistent ARPES rate, shown also as the black solid line in Fig. 3 (a). The green dot-dashed curve is the ordi- 


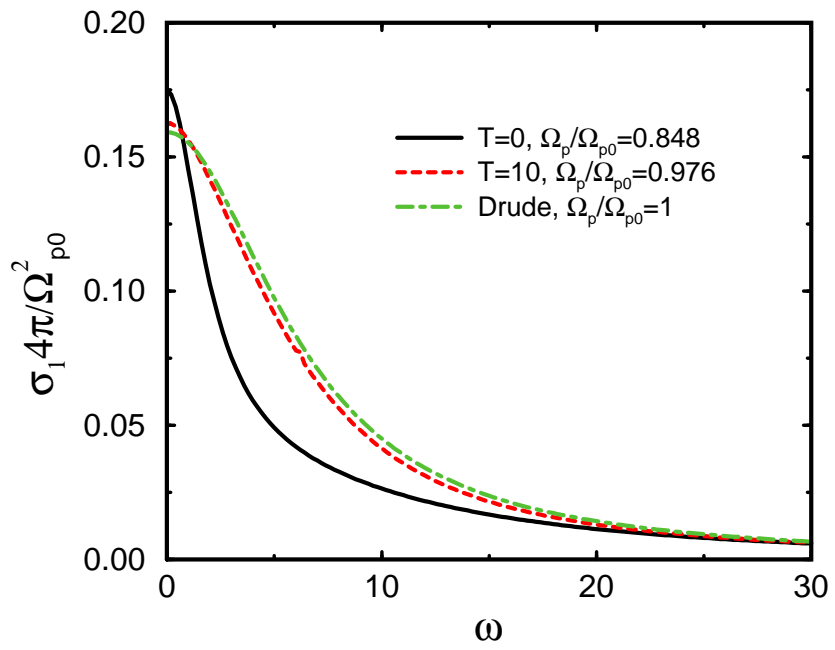

FIG. 5: Real part of optical conductivity at different temperatures in the Lorentzian-EDOS model shown in Eq. (21). We used the same $a_{1}, a_{2}, s$ and $\gamma_{0}$ as in Fig. 3 .

nary Drude for comparison. The red dashed curve shows how the solid curve evolves with increasing temperature. It represents results of $\sigma_{1}(\omega)$ vs $\omega$ with non-selfconsistent self energy for temperature $T=10$ in units of $\gamma_{0}$ which is related to the quasiparticle scattering rate. It is clear that the evolution is towards restoring the curve to its simple Drude value as can be expected when temperature or impurity effects are sufficiently strong that they wash out the energy dependence in $N(\epsilon)$. Note that for the case considered here, the energy scale for the structure in $N(\epsilon)$ is 10 in our units and this scale is of the same order as the temperature. Similar smearing effects are expected when impurity scattering is increased sufficiently that the impurity scattering rate becomes comparable to the energy scale of the structure in $N(\epsilon)$. In this case we do not show a curve analogous to Fig. 同, but instead we show the change in the optical oscillator strength under the curve, i.e., the plasma frequency. Before presenting these results we point out that in Fig. 5 at $T=0$ the plasma frequency $\Omega_{p} / \Omega_{p 0}=0.848$, while at $T=10$ it has moved up to 0.976 close to the simple Drude case, so that effects of energy dependence in $N(\epsilon)$ are pretty well washed out in $\Omega_{p}$ as they are in the full $\sigma_{1}(\omega)$ vs $\omega$ curve. In Fig. 6 we present equivalent results for the impurity scattering and compare with temperature. What is shown is the plasma frequency as a function of impurity scattering rate (or temperature). The red dashed and blue dot-dashed lines are for a peak in $N(\epsilon)$, and the black solid and magenta long-dashed lines for a valley at the Fermi energy. We see that temperature and impurity have very similar effects on the plasma frequency and that when the scale on the horizontal axis is of the order of the scale defining the structure in $N(\epsilon)$, we recover in both cases the Drude plasma frequency as we expected.

The above theoretical calculations apply to materials in which a pseudogap exists in the electronic band struc-

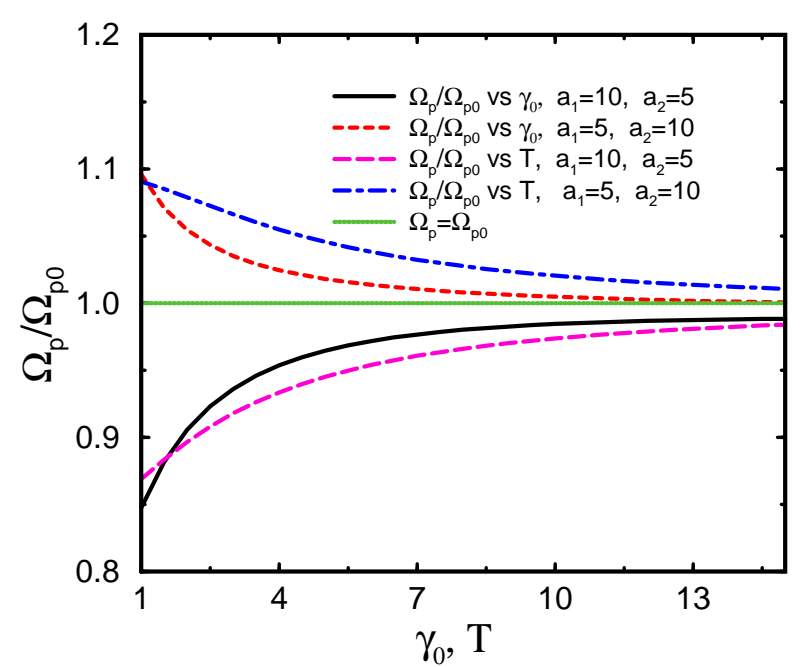

FIG. 6: Plasma frequency as a function of the impurity potential $\gamma_{0}$ and temperature $T$. We used $s=5 \pi$.

ture. The experiments need to be done at relatively low temperatures where the electrons are dominantly scattered by impurities and the systems must remain in the normal state. The underdoped cuprates exhibit a pseudogap of size $300 \mathrm{~K}$ or so. This pseudogap has its origin in many-body effects as we mentioned in the Introduction. Nevertheless, in as much as the effective EDOS around the Fermi surface is depressed, our results should give some qualitative guidance about the physical consequences of such a EDOS depression. Of course, in the cuprates a superconducting transition occurs, but $T_{c}$ can be low, of order $20 \mathrm{~K}$. In this intermediate temperature regime, however, the inelastic scattering due to electronic interaction may already be significant, which complicates the comparison. Disordering in the $\mathrm{CuO}_{2}$ planes, such as replacing copper sites by nickel, in the underdoped high$T_{c}$ cuprates, will efficiently enhance the elastic scattering, so that it becomes dominant. Alternatively, a magnetic field can be used to quench the superconductivity, enabling measurements at much lower temperatures.

\section{OPTICAL CONDUCTIVITY IN "STEP MODEL"}

Another EDOS model which allows us to get simple analytical results and also helps in developing insight into the effect of $N(\epsilon)$ on $\sigma(\omega)$ is the so-called step model,

$$
\frac{N(\epsilon)}{N_{b}}= \begin{cases}h, & |\epsilon|<E_{g} \\ 1, & |\epsilon|>E_{g}\end{cases}
$$

The non-selfconsistent self energy from Eq. (15) reads

$$
\begin{aligned}
& \Sigma_{1 \mathrm{nsc}}(\omega)=(1-h) \gamma_{0} \ln \left|\frac{\omega-E_{g}}{\omega+E_{g}}\right|, \\
& g_{\mathrm{nsc}}(\omega)=\pi \gamma_{0} \frac{N(\omega)}{N_{b}}= \begin{cases}\pi \gamma_{0} h, & |\omega|<E_{g} \\
\pi \gamma_{0}, & |\omega|>E_{g}\end{cases}
\end{aligned}
$$


The selfconsistent self energy from Eq. (16) is determined from the following equations

$$
\begin{aligned}
\Sigma_{1 \mathrm{sc}}(\omega)= & (1-h) \frac{\gamma_{0}}{2} \ln \left[\frac{\left(\omega_{0}-E_{g}\right)^{2}+g_{s c}^{2}(\omega)}{\left(\omega_{0}+E_{g}\right)^{2}+g_{s c}^{2}(\omega)}\right] \\
g_{\mathrm{sc}}(\omega)=\gamma_{0} & \left\{\pi-(1-h)\left[\arctan \left(\frac{\omega_{0}+E_{g}}{g_{\mathrm{sc}}(\omega)}\right)\right.\right. \\
& \left.\left.-\arctan \left(\frac{\omega_{0}-E_{g}}{g_{\mathrm{sc}}(\omega)}\right)\right]\right\}
\end{aligned}
$$

where $\omega_{0}=\omega-\Sigma_{1 \mathrm{sc}}(\omega)$. Eqs. (37) and (38) need to be solved numerically.

To calculate $\sigma_{1}(\omega)$ in Eq. (13), we can first integrate over $\epsilon$. Inserting Eq. (34) into Eq. (13), we find that

$$
\begin{aligned}
\sigma_{1}(\omega) & =\frac{\Omega_{p 0}^{2}}{4 \pi} \frac{1}{\pi} \int_{-\omega}^{0} \frac{d x}{\omega}\left\{\frac{1}{g(x)} \frac{\pi(\tilde{g}+1)}{(\tilde{g}+1)^{2}+\left(x_{0}-\tilde{x}\right)^{2}}\right. \\
& \left.-(1-h) \mathcal{F}^{(\mathrm{re})}\left(\tilde{E}_{g}, \tilde{g}, x_{0}, \tilde{x}\right)\right\}
\end{aligned}
$$

where $\mathcal{F}^{(\mathrm{re})}$ is found in Appendix A, $\tilde{g}=g(x+\omega) / g(x)$, $x_{0}=\left[x-\Sigma_{1}(x)\right] / g(x), \tilde{x}=\left[x+\omega-\Sigma_{1}(x+\omega)\right] / g(x)$, and $\tilde{E}_{g}=E_{g} / g(x)$.

In Fig. 7 we show the numerical results that we have obtained in the step model. Fig. 7 (a) gives the quasiparticle scattering rate $g(\omega)$ vs $\omega$ for four different cases. The green dot-dashed line is the non-selfconsistent result for $g(\omega)$ in the semiconductor-like model, i.e., $h=0$. As indicated in Eq. (36), $g(\omega)$ is exactly zero until $\omega=10$ and jumps to $\pi$ for $\omega>10$. This curve is for comparison with the full selfconsistent quasiparticle scattering rate obtained from iterating to convergence Eqs. (37) and (38). The numerical results are the blue long-dashed curve. We see a sharp scattering edge at $\Omega_{0} \simeq 6.1$ below which the scattering rate is zero, so that now the sharp onset has moved to lower energy, as compared to the non-selfconsistent case. Note that $g(\omega)$ is proportional to the fully renormalized (from impurity scattering) EDOS $\tilde{N}(\omega)$ as defined before and this shows smearing of the step function edge that existed in the non-selfconsistent case. The impurity scattering also accounts for the reduction in scattering above $\omega=10$. In fact, there is a sum rule on $\tilde{N}(\omega)$ so that the area under each curve, selfconsistent and non-selfconsistent, remains unchanged. So, the increase in fully renormalized EDOS below $\omega=10$ is fully compensated for at larger $\omega$. This also holds for the second set of two curves. In this case $h=0.1$ instead of being zero. The red dashed curve gives the non-selfconsistent results while the black solid curve is for the selfconsistent case. In this case, there is always a finite EDOS at all frequencies, but it still rises sharply at $\omega=10$ in the non-selfconsistent case and at $\omega \simeq 6.1$ in the selfconsistent calculations. However, as compared with the $h=0$ case, the rise is not sharp. There is some rounding of the edge. Conservation of states applies however.

The corresponding results for the real part of the conductivity $\sigma_{1}(\omega)$ vs $\omega$ are shown in Fig. 7 (b). We see, as
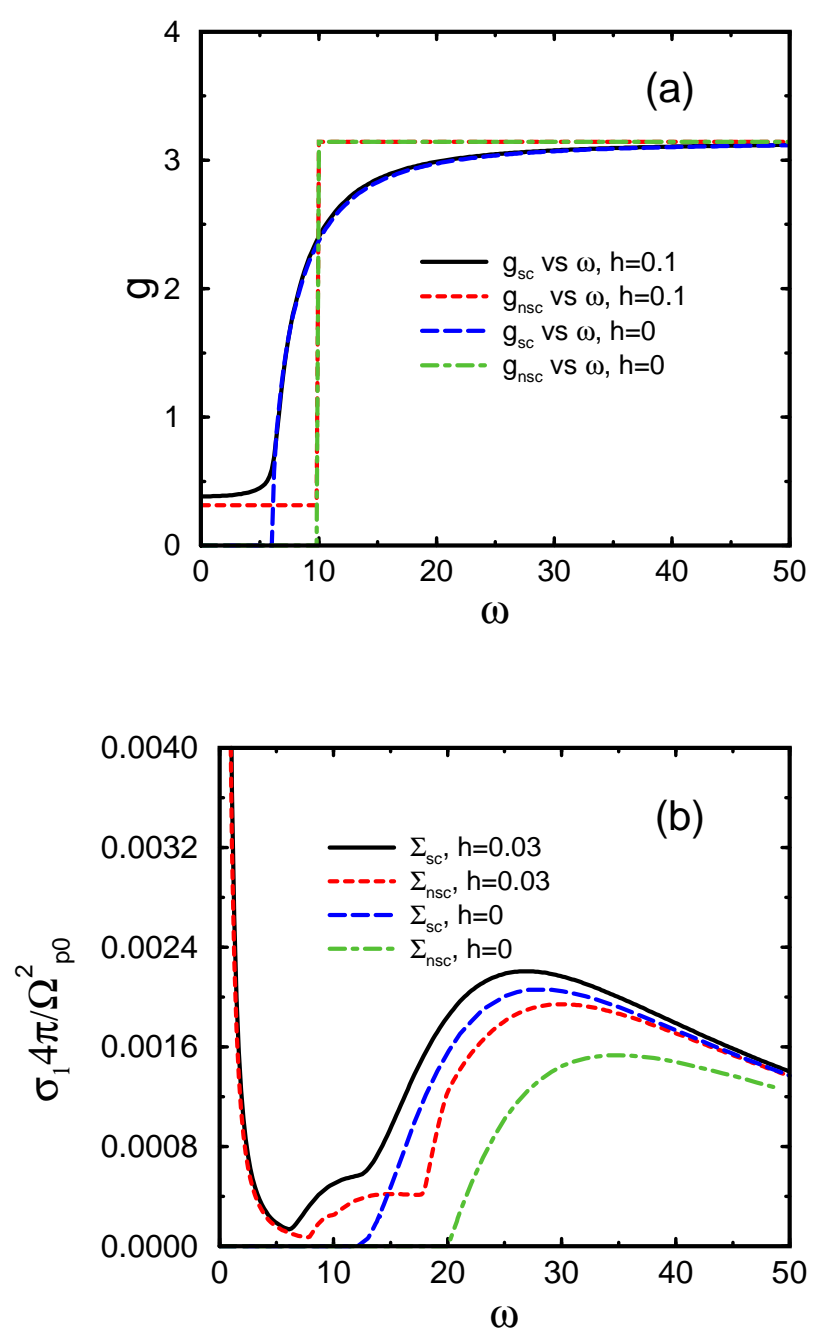

FIG. 7: (a) ARPES rate and (b) Real part of the optical conductivity, as functions of frequency in the step model shown in Eq. (34). We used $E_{g}=10$ and $\gamma_{0}=1$.

we expected, that for the pure semiconductor-like model, there is no optical absorption until $\omega=2 E_{g}=20$ in the non-selfconsistent case (green dot-dashed curve). This also holds for the selfconsistent case (blue long-dashed curve), but now the edge has moved to $\omega=2 \Omega_{0} \simeq 12.2$. The other two curves are metallic in nature because now $h=0.03 \neq 0$. The finite value of $h$ at small $\omega$ guarantees that the response in this region is metallic-like and we see a sharp Drude-like peak centered around $\omega=0$. At higher energy the semiconductor-like behavior of the two previous curves remains although the main rise in the conductivity has been shifted to lower $\omega$ as compared with $\omega=20$ and 12.2 respectively for non-selfconsistent and selfconsistent case with $h=0$. Between the Drude response at small $\omega$ and semiconducting-like response at high $\omega$ the curve gets filled in and shows sharp structures corresponding to the sharp step assumed to exist in the initial $\operatorname{EDOS} N(\epsilon)$. 
We next contrast more sharply the two qualitatively different limits discussed above using an analytical method to get simple although limited results.

Case 1. $h=0$ : Semiconducting-like behavior.

In this case, $g(\omega)=0$ for $\omega<\Omega_{0}$. (Note that for the non-selfconsistent ARPES rate, $\Omega_{0}=E_{g}$, and for the selfconsistent ARPES rate, $\Omega_{0}<E_{q}$.)

Now we look at $\sigma_{1}(\omega)$ in Eq. (13). Noting that $-\omega<$ $x<0$, we obtain:

i) For $0<\omega<\Omega_{0}$, both $A(\epsilon, x)$ and $A(\epsilon, x+\omega)$ are zero, leading to $\sigma_{1}(\omega)=0$;

ii) For $\Omega_{0}<\omega<2 \Omega_{0}, A(x+\omega)=0$ for $-\omega<x<$ $-\Omega_{0}$, while $A(x)=0$ for $-\Omega_{0}<x<0$. Therefore, $\sigma_{1}(\omega)=0$.

To conclude, $\sigma_{1}(\omega)=0$ for $\omega<2 \Omega_{0}$.

Case 2. $h=0^{+}$: Metallic-like behavior.

In this case, we expect to see the Drude peak at small $\omega$. This can be nicely shown in the dc conductivity. By substituting Eq. (34) into Eq. (14) we obtain

$$
\begin{gathered}
\sigma(0)=\frac{\Omega_{p 0}^{2}}{4 \pi} \frac{1}{\pi} \frac{1}{g(0)}\left\{( h - 1 ) \left[\frac{E_{g} g(0)}{E_{g}^{2}+g^{2}(0)}\right.\right. \\
\left.\left.+\arctan \left(\frac{E_{g}}{g(0)}\right)\right]+\frac{\pi}{2}\right\} .
\end{gathered}
$$

It is clear that for $h=1, \mathrm{Eq}$. (40) recovers the Drude result, $\sigma^{\text {(Drude) }}(0)=\left(\Omega_{p 0}^{2} / 4 \pi\right) / 2 g(0)$. In the limit of $h \rightarrow$ $0^{+}$, we get from Eq. (40) that

$$
\sigma^{\left(h=0^{+}\right)}(0) \simeq \frac{\Omega_{p 0}^{2}}{4 \pi} \frac{h}{2 g(0)} .
$$

For the non-selfconsistent case, $g_{\mathrm{nsc}}(0)=\pi \gamma_{0} h$, thus

$$
\sigma_{\mathrm{nsc}}^{\left(h=0^{+}\right)}(0) \simeq \frac{\Omega_{p 0}^{2}}{4 \pi} \frac{1}{2 \pi \gamma_{0}}=\sigma^{(\text {Drude })}(0) .
$$

For the selfconsistent case, however, Eq. (38) produces, in linear order of $h, g_{\mathrm{sc}}(0) \simeq \pi \gamma_{0} h /\left(1-2 \gamma_{0} / E_{g}\right)$ (for $\left.2 \gamma_{0}<E_{g}\right)$, leading to

$$
\sigma_{\mathrm{sc}}^{\left(h=0^{+}\right)}(0) \simeq \frac{\Omega_{p 0}^{2}}{4 \pi} \frac{1}{2 \pi \gamma_{0}}\left(1-\frac{2 \gamma_{0}}{E_{g}}\right),
$$

which is different from the corresponding Drude value.

We can also compare the above results obtained in the EDOS-step model for a finite $h$ value with those obtained in the EDOS model with one Lorentzian form, $N(\epsilon) / N_{b}=1-(s / \pi) a_{2} /\left(a_{2}^{2}+\epsilon^{2}\right)$. We find that in the Lorentzian-form model, $\sigma_{1}(\omega)$ shows a sharp Drude-like peak at low frequencies and semiconducting-like rise at around $\omega \sim a_{2}$, which are similar to what is shown in the solid black curve in Fig. 7. However, since both the EDOS and the ARPES rate are smooth in this model, the conductivity curve is always smoothly evolving as a function of frequency, and the semiconducting-like rise can never be as sharp as in the step model.

\section{CONCLUSION}

We have studied the effect, energy dependence in the EDOS around the Fermi energy, has on the frequency dependent conductivity, and the derived optical scattering rate. Only elastic impurity scattering is accounted for. To keep the mathematics simple, we employ for $N(\epsilon)$ a constant background modified by two Lorentzians both centered at the Fermi energy so as to provide particlehole symmetry. One adds states the other subtracts, so that the sum over all states is not modified. One of the integrals defining the conductivity in terms of the electron self energy $\Sigma(\omega)$ can be done analytically. This greatly simplifies the numerical work and allows us to find certain important limits in closed form. The conductivity is modified in two important ways. One comes from the energy-dependent EDOS factor which enters when a sum is to be carried out over all the electrons that contribute to the current. We referred to this as the initial state factor. The second factor comes from modification of the quasiparticle scattering rate which is no longer constant and in fact becomes proportional to the selfconsistent EDOS $\tilde{N}(\epsilon)$. This quantity differs from $N(\epsilon)$ in that it accounts for the smearing of the EDOS brought about by the impurities. It is to be computed from the selfconsistent self energy equations. Both factors have a profound effect on the conductivity, but their individual contribution cannot easily be separated. For a depression in $N(\epsilon)$ at the Fermi energy, the first factor reduces the real part of the conductivity at and around $\omega=0$ (dc conductivity) effectively, making the resulting optical scattering rate appear larger than its value in the pure Drude model, i.e., the width of the curve at half maximum is increased. On the other hand, the second factor acts in the opposite manner. Since the quasiparticle scattering rate becomes proportional to $\tilde{N}(\omega)$, this quantity now acquires a frequency dependence which, in the model under consideration, reduces the scattering rate at and around $\omega=0$, because $\tilde{N}(\omega)$ is less than the constant background value there. This has the effect of increasing the height of the Drude at small $\omega$ and making it narrower as compared to the constant case. The net result of both effects in the selfconsistent case is to produce a curve for $\sigma_{1}(\omega)$ vs $\omega$ which is reduced below the pure Drude at small $\omega$. The corresponding optical scattering rate is depressed at small $\omega$ below its constant Drude value and then becomes larger at higher $\omega$. This mimics the variation in the quasiparticle scattering rate which is proportional to $\tilde{N}(\omega)$, but there are important differences. In particular, while a sum rule applies to the ARPES scattering rate which reflects quite directly the sum rule on the EDOS, the optical scattering rate displays no such property. It is clear then that when there is important energy dependence in the EDOS at the Fermi energy, optical and quasiparticle scattering rates are not as simply related as in the Drude model. This complicates the process of extracting microscopic information from optical scattering rate data. What one ultimately 
wants is detail information on the self energy.

There are several other complications that arise that need to be commented upon. The plasma frequency which gives the optical oscillator strength, i.e., the area under the real part of the conductivity, becomes dependent on temperature and on impurity concentration. We show that the plasma frequency depends on a range of states around the Fermi energy with the energy scale associated with this range given by the impurity scattering rate. It is not just its value at the Fermi surface which matters. As the impurity rate is changed, the range of important values of $N(\epsilon)$ is also changed and so is $\Omega_{p}$. In the models considered this can be a significant effect. Temperature can also smear out the region in energy around the Fermi surface and so also impact on the value of $\Omega_{p}$. As temperature is increased, we find that the EDOS effects become gradually less important and the entire curve for $\sigma_{1}(\omega)$ vs $\omega$ moves towards the Drude form with constant background only in $N(\epsilon)$. Another result of interest is that the region in energy most important in determining the plasma frequency is different from that determining the dc conductivity, so that these two quantities will not respond in the same way with change in impurity content.

Finally we have considered a step function model for $N(\epsilon)$ around the Fermi surface with $N(\epsilon)=h N_{b}$ for $0<$ $\epsilon<E_{g}$, and $N(\epsilon)=N_{b}$ for $\epsilon>E_{g}$, where $E_{g}$ is a gap energy. For $h=0$, this is a semiconductor-like model which leads directly to zero conductivity in the range $0 \leq$ $\omega \leq 2 \Omega_{0}\left(\Omega_{0}=E_{g}\right.$ for non-selfconsistent ARPES rate, and $\Omega_{0}<E_{g}$ for selfconsistent ARPES rate). We find that for finite $h$, however small it may be, the situation is radically different and an intrinsically metallic behavior is always obtained. At small $\omega$, there is a very narrow Drude-like peak followed by a depression, and at higher energies $\omega \geq \Omega_{0}$ a semiconductor-like behavior is again observed. The value of the dc conductivity is unchanged from its value with $N(\epsilon)=N_{b}$ everywhere provided $h \ll$ 1. Here we have described only the case when the self energy is treated in a first iteration. We find however that the situation shows no qualitative change when a self consistent theory is considered except for the important difference that the gap $\Omega_{0}$ is reduced by the interactions and the dc conductivity has a correction linear in $\gamma_{0} / E_{g}$.

\section{Acknowledgments}

We thank E. J. Nicol for helpful discussion. This work is partially supported by the Natural Science and Engineering Research Council of Canada and by the Canadian Institute for Advanced Research.

\section{APPENDIX A: EXPRESSIONS FOR $\mathcal{F}^{(\mathrm{re})}, \mathcal{F}^{(\mathrm{im})}$ AND $\mathcal{F}_{0}^{(\mathrm{im})}$ IN EQS. (32) AND (33)}

As mentioned in Sec.III.B, in the case of a constant $N(\epsilon)=N_{b}$ and energy-dependent ARPES rate, we can first integrate over $\epsilon$. The resulting optical conductivity at $T=0$ is shown in Eqs. (32) and (33), where

$$
\begin{aligned}
& \mathcal{F}^{(\mathrm{re})}\left(\tilde{D}, g, x_{0}, x\right)=d_{0}\left[g\left(y^{2}+g^{2}-1\right) f_{1}+\left(y^{2}-g^{2}+1\right) f_{2}-g y f_{3}\right], \\
& \mathcal{F}^{(\mathrm{im})}\left(\tilde{D}, g, x_{0}, x\right)=\frac{d_{0}}{2}\left[-2 y\left(y^{2}+g^{2}+1\right) f_{1}+4 g y f_{2}+\left(1-g^{2}+y^{2}\right) f_{3}\right], \\
& \mathcal{F}_{0}^{(\mathrm{im})}\left(\tilde{D}, x_{0}\right)=4 x_{0} \tilde{D}\left[\left(x_{0}-\tilde{D}\right)^{2}+1\right]^{-1}\left[\left(x_{0}+\tilde{D}\right)^{2}+1\right]^{-1},
\end{aligned}
$$

with $d_{0}=\left[(g+1)^{2}+y^{2}\right]^{-1}\left[(g-1)^{2}+y^{2}\right]^{-1}, y=x_{0}-x, f_{1}=\arctan \left(\tilde{D}+x_{0}\right)+\arctan \left(\tilde{D}-x_{0}\right), f_{2}=\arctan [(\tilde{D}+$ $x) / g]+\arctan [(\tilde{D}-x) / g]$, and $f_{3}=\ln \left\{\left[\left(\tilde{D}-x_{0}\right)^{2}+1\right] /\left[\left(\tilde{D}+x_{0}\right)^{2}+1\right]\right\}+\ln \left\{\left[(\tilde{D}+x)^{2}+g^{2}\right] /\left[(\tilde{D}-x)^{2}+g^{2}\right]\right\}$.

\section{APPENDIX B: OPTICAL CONDUCTIVITY FOR ENERGY-DEPENDENT ARPES RATE IN THE LORENTZIAN DOS MODEL}

For the EDOS model with Lorentzian forms shown in Eq. 21) and the energy-dependent ARPES rate, we can first integrate $\epsilon$ to simplify the numerical work. The resulting conductivity reads

$$
\begin{aligned}
& \sigma_{1}(\omega)=\sigma_{1}^{(\mathrm{cons})}(\omega)+\delta \sigma_{1}\left(\omega, a_{1}\right)-\delta \sigma_{1}\left(\omega, a_{2}\right), \\
& \sigma_{2}(\omega)=\sigma_{2}^{(\mathrm{cons})}(\omega)+\delta \sigma_{2}\left(\omega, a_{1}\right)-\delta \sigma_{2}\left(\omega, a_{2}\right),
\end{aligned}
$$

where $\sigma_{1}^{(\mathrm{cons})}$ and $\sigma_{2}^{(\mathrm{cons})}$ are shown in Eqs. (32) and (33), and

$$
\delta \sigma_{1}(\omega, a)=\frac{\Omega_{p 0}^{2}}{4 \pi} \frac{s}{2 \pi} \int_{-\omega}^{0} \frac{d x}{\omega}\left[\frac{x_{0} x_{1}+g_{a+} g_{1 a-}}{\left(x_{0}^{2}+g_{a+}^{2}\right)\left(x_{1}^{2}+g_{1 a-}^{2}\right)}+\frac{x_{0} x_{2}-g_{a+} g_{2 a+}}{\left(x_{0}^{2}+g_{a+}^{2}\right)\left(x_{2}^{2}+g_{2 a+}^{2}\right)}\right]
$$




$$
+\frac{\Omega_{p 0}^{2}}{4 \pi} \frac{s a}{\pi} \int_{-\omega}^{0} \frac{d x}{\omega} \frac{2\left(x_{1}-x_{0}\right) x_{1} g_{1}+\left(g_{0}+g_{1}\right)\left(a^{2}+x_{1}^{2}-g_{1}^{2}\right)}{\left[\left(x_{1}-x_{0}\right)^{2}+\left(g_{0}+g_{1}\right)^{2}\right]\left[\left(a^{2}+x_{1}^{2}-g_{1}^{2}\right)^{2}+4 x_{1}^{2} g_{1}^{2}\right]}
$$

$$
\begin{gathered}
\delta \sigma_{2}(\omega, a)=\frac{\Omega_{p 0}^{2}}{4 \pi} \frac{s}{2 \pi} \int_{-\infty}^{0} \frac{d x}{\omega}\left[\frac{x_{2} g_{a-}-x_{0} g_{2 a+}}{\left(x_{0}^{2}+g_{a-}^{2}\right)\left(x_{2}^{2}+g_{2 a+}^{2}\right)}+\frac{x_{1} g_{a+}-x_{0} g_{1 a-}}{\left(x_{0}^{2}+g_{a+}^{2}\right)\left(x_{1}^{2}+g_{1 a-}^{2}\right)}+\frac{x_{0} g_{1 a+}+x_{1} g_{a+}}{\left(x_{0}^{2}+g_{a+}^{2}\right)\left(x_{1}^{2}+g_{1 a+}^{2}\right)}\right. \\
\left.+\frac{x_{0} g_{2 a+}+x_{2} g_{a+}}{\left(x_{0}^{2}+g_{a+}^{2}\right)\left(x_{2}^{2}+g_{2 a+}^{2}\right)}-\frac{4 x g_{a+}}{\left(x^{2}+g_{a+}^{2}\right)^{2}}\right]-\frac{\Omega_{p 0}^{2}}{4 \pi} \frac{s a}{\pi} \int_{-\infty}^{0} \frac{d x}{\omega}\left\{\frac{\left(x_{0}-x_{2}\right)\left(a^{2}+x_{0}^{2}-g_{0}^{2}\right)-2 x_{0} g_{0}\left(g_{0}+g_{2}\right)}{\left[\left(x_{0}-x_{2}\right)^{2}+\left(g_{0}+g_{2}\right)^{2}\right]\left[\left(a^{2}+x_{0}^{2}-g_{0}^{2}\right)^{2}+4 x_{0}^{2} g_{0}^{2}\right]}\right. \\
\left.-\frac{\left(x_{1}-x_{0}\right)\left(a^{2}+x_{1}^{2}-g_{1}^{2}\right)-2 x_{1} g_{1}\left(g_{0}+g_{1}\right)}{\left[\left(x_{1}-x_{0}\right)^{2}+\left(g_{0}+g_{1}\right)^{2}\right]\left[\left(a^{2}+x_{1}^{2}-g_{1}^{2}\right)^{2}+4 x_{1}^{2} g_{1}^{2}\right]}\right\},
\end{gathered}
$$

with $x_{0}=x-\Sigma_{1}(x), x_{1}=x+\omega-\Sigma_{1}(x+\omega), x_{2}=x-\omega-\Sigma_{1}(x-\omega), g_{0}=g(x), g_{1}=g(x+\omega), g_{2}=g(x-\omega)$, $g_{a \pm}=g_{0} \pm a, g_{1 a \pm}=g_{1} \pm a, g_{2 a \pm}=g_{2} \pm a$.

1 A. V. Puchkov, D. N. Basov and T. Timusk, J. Phys.: Cond. Matt. 8, 10049 (1996).

2 T. Timusk and B. Statt. Rep. Prog. Phys. 62, 61 (1999).

${ }^{3}$ C. M. Varma, Int. J. Mod. Phys. 3, 2083 (1989).

${ }^{4}$ C. M. Varma, P. B. Littlewood, S. Schmitt-Rink, E. Abrahams, and A. E. Ruckenstein, Phys. Rev. Lett. 63, 1996 (1989); ibid 64, 497 (1990).

${ }^{5}$ E. Abrahams and C. M. Varma, cond-mat/0003135 (unpublished).

${ }^{6}$ F. Marsiglio and J. P. Carbotte, Aust. J. Phys. 50, 975 (1997).

7 F. Marsiglio and J. P. Carbotte, Aust. J. Phys. 50, 1011 (1997).

${ }^{8}$ R. E. Walstedt, W. W. Warren, Jr., R. F. Bell, R. J. Cava, G. P. Espinosa, L. F. Schneemeyer, and J. V. Waszczak, Phys. Rev. B 41, 9574 (1990).

${ }^{9}$ W. W. Warren, Jr., R. E. Walstedt, G. F. Brennert, R. J. Cava, R. Tycko, R. F. Bell, and G. Dabbagh, Phys. Rev. Lett. 62, 1193 (1989).

10 H. Takagi, B. Batlogg, H. L. Kao, J. Kwo, R. J. Cava, J. J. Krajewski, and W. F. Peck, Jr., Phys. Rev. Lett. 69, 2975 (1992).

11 T. Ito, K. Takenaka, and S. Uchida, Phys. Rev. Lett. 70, 3995 (1993).

12 J. W. Loram, K. A. Mirza, J. R. Cooper and W. Y. Liang, J. Supercond. 7, 243 (1994).

13 J. W. Loram, K. A. Mirza, J. R. Cooper and J. L. Tallon, Physica C 282-287, 1405 (1997).

${ }^{14}$ J. W. Loram, K. A. Mirza, J. R. Cooper N. Athanassopoulou and W. Y. Liang, in Proc. 10th HTC Anniversary Workshop on Physics, Materials and Applications, edited by B. Batlogg et al., (Singapore, World Scientific (1996)) p341.

${ }^{15}$ C. C. Homes, T. Timusk, R. Liang, D. A. Bonn, and W. N. Hardy, Phys. Rev. Lett. 71, 1645 (1993).

${ }^{16}$ Ch. Renner, B. Revaz, J.-Y. Genoud, K. Kadowaki, and O. Fischer, Phys. Rev. Lett. 80, 149 (1998).

17 A. G. Loeser, Z. X. Shen, D. S. Dessau, D. S. Marshall, C. H. Park, P. Fournier, and A. Kapitulnik, Science 273, 325 (1996).
18 H. Ding, T. Yobaya, J. C. Campuzano, T. Takahashi, M. Randeria, M. R. Norman, T. Mochiku, K. Kadowaki, J. Giapinzakis, Nature 382, 51 (1996).

19 J. M. Harris, Z.-X. Shen, P. J. White, D. S. Marshall, M. C. Schabel, J. N. Eckstein, and I. Bozovic, Phys. Rev. B 54, 15665 (1996).

20 P. W. Anderson, Science 235, 1196 (1987).

21 P. A. Lee and N. Nagaosa, Phys. Rev. B 46, 5621 (1992).

22 P. A. Lee and X. G. Wen, Phys. Rev. Lett. 78, 4111 (1997).

23 V. J. Emery, S. A. Kivelson, and O. Zachar, Phys. Rev. B 56,6120 (1997).

24 V. J. Emery and S. A. Kivelson, Nature (London) 374, 434 (1995).

25 Qijin Chen, Ioan Kosztin, Boldizsár Jankó, and K. Levin, Phys. Rev. Lett. 81, 4708 (1998).

26 S. Chakravarty, R. B. Laughlin, D. K. Morr, and C. Nayak, Phys. Rev. B 63, 094503 (2001).

27 F. Marsiglio, J. P. Carbotte and E. Schachinger, Phys. Rev. B 65, 014515 (2002).

28 D. N. Basov, E. J. Singley, S. V. Dordevic, condmat/0103507.

${ }^{29}$ G. D. Mahan, Many-Particle Physics (Plenum Press, New York and London, 1993).

30 B. M. Klein, D. A. Papaconstantopoulos, and L. L. Boyer, Superconductivity in $d$ - and $f$-Band Metals, edited by Suhl and M. B. Maple (Academic Press, New York, 1980) p455.

31 P. Horsch and H. Rietschel, Z. Phys. B 27, 153 (1977).

32 S. J. Nettel and H. Thomas, Solid State Commun. 21, 683 (1977).

33 S. G. Lie and J. P. Carbotte, Solid State Commun. 26, 511 (1978).

34 M. Weger and I. B. Goldberg, Solid State Physics, edited by M. Ehrenreich, F. Seitz, and D. Turnbull (Academic Press, New York, 1973), Vol. 28, p. 1.

35 W. E. Pickett, Phys. Rev. B 21, 3897 (1980).

36 B. Mitrović and J. P. Carbotte, Can. J. Phys. 61, 758 (1983); ibid. 61, 784 (1983); ibid. 61, 872 (1983).

37 B. Mitrović, Ph.D Thesis, McMaster (1981). 